\title{
Synchronous Invasive Ductal Carcinoma and Mucosa-Associated Lymphoid Tissue (MALT) Lymphoma of the Breast
}

\author{
Jung Hee Byon ${ }^{1}$ and Eun Jung Choi ${ }^{1}$ \\ ${ }^{1}$ Jeonbuk National University Hospital
}

March 3, 2022

\begin{abstract}
We described a rare presentation of synchronous extranodal marginal zone lymphoma of mucosa-associated lymphoid tissue (MALT) lymphoma and invasive ductal carcinoma of the breast. This case highlights that one should keep an open mind to the possibility of synchronous multiple primary neoplasms in patients with carcinoma.

\section{Synchronous Invasive Ductal Carcinoma and Mucosa-Associated Lymphoid Tissue (MALT) Lymphoma of the Breast}

Jung Hee Byon, M.D., Ph.D. ${ }^{1}$, Eun Jung Choi, M.D., Ph.D. ${ }^{1}$

Jung Hee Byon, M.D., Ph.D.

${ }^{1}$ Department of Radiology and Research Institute of Clinical Medicine of Jeonbuk National UniversityBiomedical Research Institute of Jeonbuk National University Hospital, Jeonbuk National University Medical School, 20 Geonji-ro, Deokjin-gu, Jeonju City, Jeollabuk-Do 54907, South Korea, ORCID: 0000-0002-5139552X, Email: a98038304@gmail.com

Eun Jung Choi, M.D., Ph.D.

${ }^{1}$ Department of Radiology and Research Institute of Clinical Medicine of Jeonbuk National UniversityBiomedical Research Institute of Jeonbuk National University Hospital, Jeonbuk National University Medical School, 20 Geonji-ro, Deokjin-gu, Jeonju City, Jeollabuk-Do 54907, South Korea, ORCID : 0000-0002-23394327, Email: cejcej80@hanmail.net
\end{abstract}

Reprint requests to: Eun Jung Choi, $M D$, PhD

${ }^{1}$ Department of Radiology and Research Institute of Clinical Medicine of Jeonbuk National UniversityBiomedical Research Institute of Jeonbuk National University Hospital, Jeonbuk National University Medical School 20 Geonji-ro, Deokjin-gu, Jeonju City, Jeollabuk-Do 54907, South Korea

Telephone number: +82-63-250-1150

Fax number: +82-63-272-0481

Email: cejcej80@hanmail.net

Written informed consent was obtained from the patient to publish this report in accordance with the journal's patient consent policy

Key Clinical Message 
We described a rare presentation of synchronous extranodal marginal zone lymphoma of mucosa-associated lymphoid tissue (MALT) lymphoma and invasive ductal carcinoma of the breast. This case highlights that one should keep an open mind to the possibility of synchronous multiple primary neoplasms in patients with carcinoma.

\section{Case description}

A 36-year-old woman was referred to our center with a painless palpable mass in the upper central portion of the left breast. She had no family history of breast cancer and no personal history of other malignancies. Ultrasonography (US) showed an approximately $3.5 \mathrm{x} 3 \mathrm{~cm}$ irregularly shaped, indistinct, hypoechoic mass with multiple ipsilateral enlarged axillary lymph nodes (Fig. 1A). Preoperative magnetic resonance imaging (MRI) showed an approximately $3.9 \times 3.5 \mathrm{~cm}$ irregular mass with heterogeneous enhancement and multiple enlarged axillary lymph nodes (Fig. 1B). On MRI, an irregular mass with heterogeneous enhancement was found on the upper inner quadrant of the right breast, and we recommended MR-guided US for this lesion (Fig. 2A). On MR-guided US, an approximately $1.4 \times 0.4 \mathrm{~cm}$ irregular-shaped and indistinct mass was detected that correlated to the MR lesion (Fig. 2B). US-guided core biopsies for both breast lesions and the left axillary lymph node were performed, and the pathologic results confirmed invasive ductal carcinoma in the left breast, suggested MALT lymphoma in the right breast, and confirmed metastatic adenocarcinoma in the left axillary lymph node. Although the synchronous occurrence of multiple neoplastic processes is uncommon, the possibility of synchronous breast carcinoma and primary breast lymphoma should be considered to avoid inaccurate staging and delays in diagnosis and to ensure good therapeutic and prognostic implications $(1$, 2).

\section{Conflict of interest}

The authors have stated that they have no conflicts of interest.

\section{Funding}

This work was supported by a National Research Foundation of Korea (NRF) grant funded by the Korean government (No.2021R1G1A1006474).

\section{Author contribution}

JH Byon and EJ Choi: contributed to the design and implementation of the research, analysis of the results, and writing of the manuscript.

\section{REFERENCES}

1. Liu W, Zhu H, Zhou X. Synchronous bilateral non-Hodgkin's diffuse large B-cell lymphoma of the breast and left breast invasive ductal carcinoma: a case report and review of literature. Int J Clin Exp Pathol. 2014;7(10):7042-8.

2. Cuff KE, Dettrick AJ, Chern B. Synchronous breast cancer and lymphoma: a case series and a review of the literature. J Clin Pathol. 2010;63(6):555-7.

\section{Figure legend}

Figure 1. Primary invasive ductal carcinoma in the left breast was demonstrated on ultrasound (US) and magnetic resonance imaging (MRI).

1A: Invasive ductal carcinoma involving the left upper breast was revealed on ultrasound as an irregularly shaped, indistinct, hypoechoic mass.

1B: Maximum intensity projection (MIP) of breast MRI showed invasive ductal carcinoma involving the left whole breast with advanced axillary lymph node metastasis.

Figure 2. Synchronous mucosa-associated lymphoid tissue (MALT) lymphoma was shown on magnetic resonance imaging (MRI) and ultrasound (US). 
2A: Irregular mass with heterogeneous enhancement was found on the upper inner quadrant of the right breast on breast MRI.

2B: On MR-guided US, an approximately $1.4 \times 0.4 \mathrm{~cm}$ irregular-shaped and indistinct hypoechoic mass was found. MALT lymphoma was diagnosed on subsequent core needle biopsy.
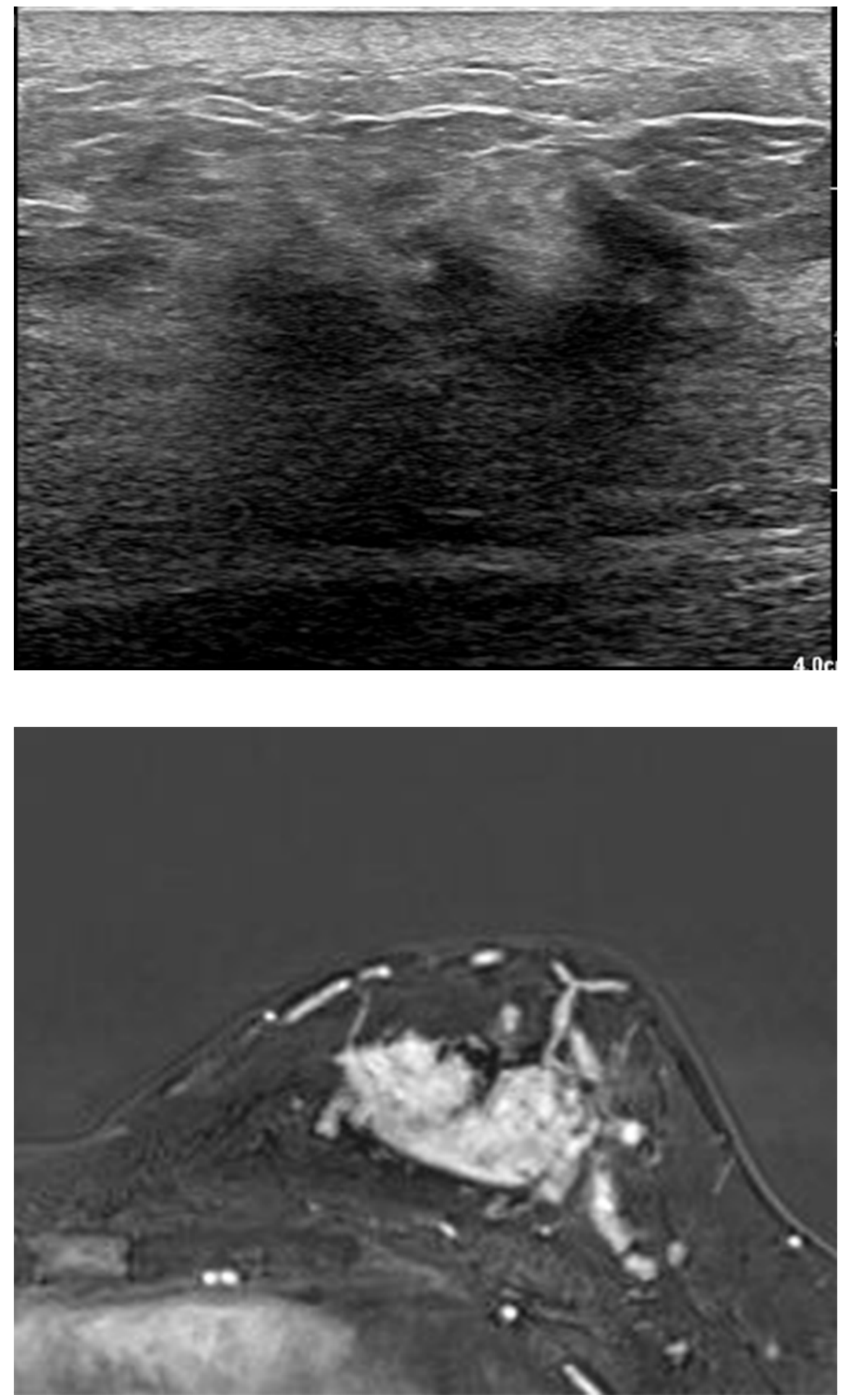

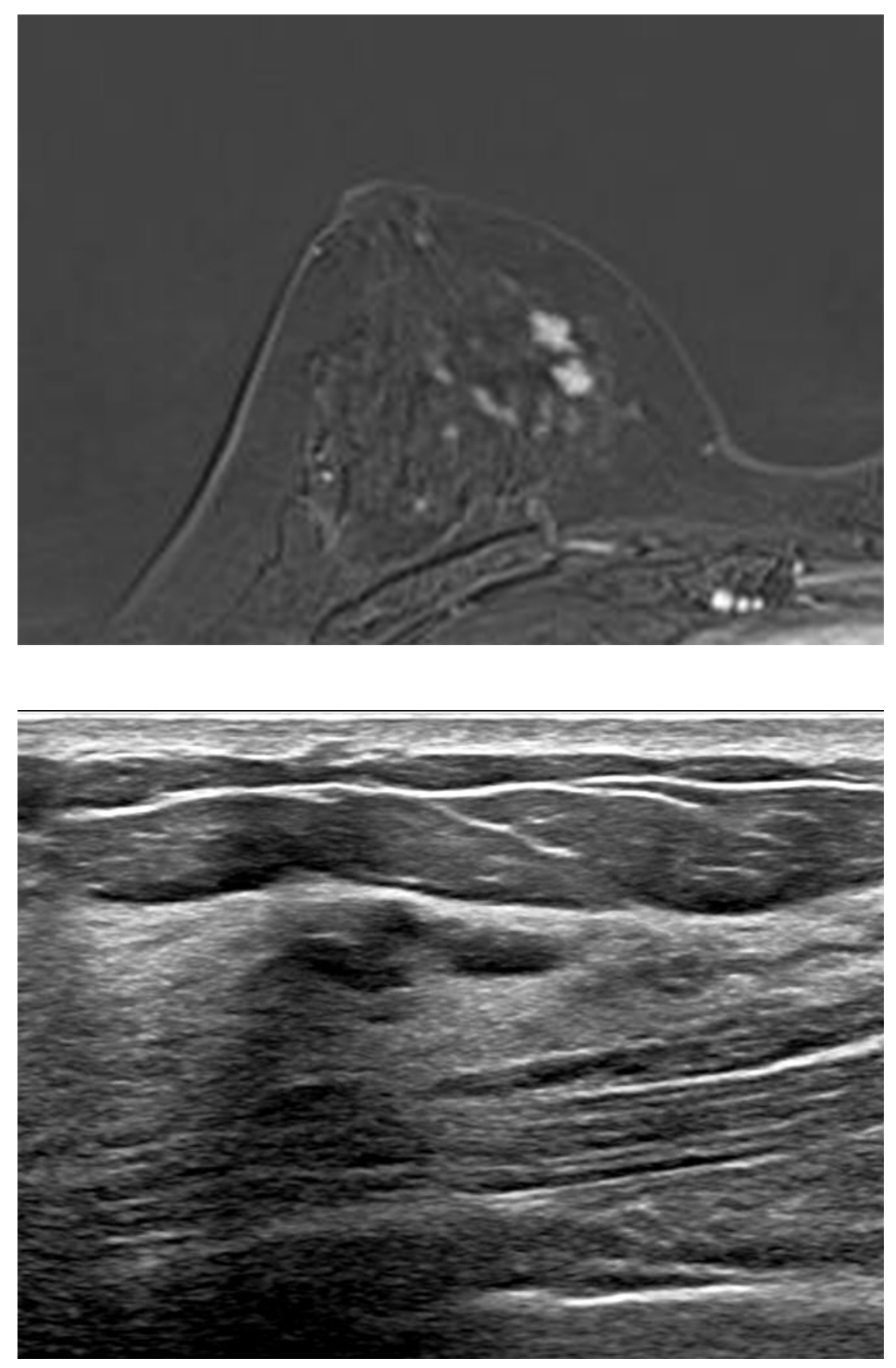